CU-TP-987

NIKHEF-00-024

YITP-00-43

UPR-900-T

\title{
Dynamical Topology Change in M Theory
}

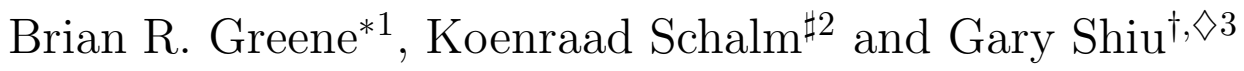 \\ * Departments of Physics and Mathematics, Columbia University \\ New York, NY 10027 \\ \# NIKHEF Theory Group \\ P.O. Box 41882 \\ Amsterdam 1009DB, The Netherlands \\ ${ }^{\dagger}$ C.N. Yang Institute for Theoretical Physics, State University of New York \\ Stony Brook, NY 11794 \\ $\diamond$ Department of Physics and Astronomy, University of Pennsylvania \\ Philadelphia, PA 1910侓
}

\begin{abstract}
We study topology change in $\mathrm{M}$ theory compactifications on Calabi-Yau three-folds in the presence of $G$ flux (the four form field strength). In particular, we discuss vacuum solutions in strongly coupled heterotic string theory in which the topology change is inevitable within a single spacetime background. For rather generic choices of initial conditions, the field equations drive the Kähler moduli outside the classical moduli space of a Calabi-Yau manifold. Consistency of the solution suggests that degenerate flop curves - just as wrapped M theory fivebranes - carry magnetic charges under the four form field strength.
\end{abstract}

\footnotetext{
${ }^{1}$ E-mail: greene@phys.columbia.edu

${ }^{2}$ E-mail: kschalm@nikhef.nl

${ }^{3}$ E-mail: shiu@insti.physics.sunysb.edu

${ }^{4}$ Address after September 1, 2000
} 


\section{Introduction}

Over the years, several works [1, 2] have established definitively that there are physically smooth processes in string theory which result in a change in the topology of spacetime. In these studies, as well as studies of topology change in M theory [3], one considers a one parameter family of vacuum solutions - a one parameter family of spacetimes - that passes from one Calabi-Yau manifold to another which is topologically distinct. The referenced works succeeded in showing that there is no obstruction to such topology change, but no dynamics was ascribed to motion through the family. In the present work, we study a variation on this theme of topology change in which dynamics does drive the evolution from one topology to another. Specifically, (a) the topology change occurs within a single (not a family of) spacetime background and (b) for generic choices of initial conditions, the dynamics (i.e., the field equations) drive us through a topology change.

To be concrete, we focus our studies on Calabi-Yau compactifications of M theory to five dimensions in the presence of $G$ flux (the four form field strength). As discussed in [5, 6, 9, 14], the effective five dimensional theory does not admit a flat space vacuum solution. Rather, the spacetime metric is warped and the solution is of the domain wall type with one of the five dimensions singled out as the transverse direction. In addition to the effective five dimensional spacetime metric, the moduli of the Calabi-Yau will generically vary along the transverse direction. In this paper, we show that there exist Calabi-Yau compactifications in which the field equations force the Kähler moduli to pass from one Kähler cone into an adjacent cone, while the overall volume of the Calabi-Yau manifold remains large. This implies that the Calabi-Yau manifold undergoes a flop transition and continues on to a topologically distinct Calabi-Yau manifold as we move along the transverse dimension.

One may think of our work as being complementary to that of [20, 21, 22] in which it was shown that in the presence of certain dyonic black holes, a Calabi-Yau with particular moduli at spatial infinity can be driven by the attractor equations through a flop transition on the way to the black hole's horizon. Here we find vacuum solutions whose structure requires topology change.

One feature of these topology changing solutions is that flop curves appear to be magnetic sources for the $G$ flux. This becomes apparent from the Bianchi identity for the four-form $G$ in the context of strongly coupled heterotic string theory (which we shall henceforth refer to as Horava-Witten theory), i.e. $\mathrm{M}$ theory on $S_{1} / \mathbb{Z}_{2}$. In this set-up, the orbifold planes provide magnetic sources for the four form $G$ and this requires a modification of the Bianchi identity by a topological source term [4]. As we will see, consistency of the topology changing solutions suggests that the zero-size flop curve provides an additional magnetic source for $G$.

While we will not pursue it in this paper, the results of the present work may have implications for the "brane world" scenario [4, 6, 10, 11, 12, 13]. Strongly coupled heterotic string theory is a rich context for the brane-world scenario in which fairly realistic low energy models can be constructed [6]. The class of models of interest, however, is substantially enlarged, as we allow the topology, and not just the metric, of the Calabi-Yau to change from one end of the world to another.

This paper is organized as follows. In Section 2, we summarize the essential results of M 
theory and Horava-Witten theory compactified on Calabi-Yau three-folds in the presence of $G$ flux. In Section 3, we give an explicit example in which the solution to the field equations inevitably drives us through a flop transition. In Section 4 , we discuss general features of topology changing solutions in Horava-Witten theory, and examine properties of $G$ flux and the Bianchi identity in such geometric backgrounds. We end with some discussion in Section 5.

After the presentation of these results by one of us [29], M. Wijnholt and S. Zhukov notified us that they had also observed that the Bianchi identity requires modification in the presence of a flop transition.

\section{Kaluza-Klein Reduction of $M$ theory on a Calabi- Yau three-fold}

Let us briefly summarize the results of the Kaluza-Klein reduction of eleven dimensional supergravity on a manifold with boundaries, that is, strongly coupled heterotic theory on a Calabi-Yau three-fold $\mathcal{M}$. (Many details can be found in the papers [6, 0, 8, 9]; we differ in approach by using a first order formalism for the field strengths, see also [23. The appropriate Bianchi identities are imposed as an additional field equation.) We concentrate here only the fields which are relevant to our subsequent discussion. Since we seek solutions with nontrivial variation of the Kähler moduli, we keep the hypermultiplet scalar $V$ (the Calabi-Yau breathing mode) which couples to the bulk potential terms, the vector multiplet scalars $b^{i}$ (the Kähler moduli) as well as the axionic field strengths $a_{i}$ and their dual potentials $\tilde{\lambda}^{i}$. The 5-dimensional action can be consistently truncated to this reduced field content leading to 6 ]

$$
\begin{array}{r}
S_{5}=-\frac{L^{6}}{2 \ell^{9}}\left[\int_{M_{5}} \sqrt{-g}\left(R+G_{i j}(b) \partial_{M} b^{i} \partial^{M} b^{j}+\frac{1}{2} V^{-2} \partial_{M} V \partial^{M} V+\lambda(\mathcal{K}-1)\right)\right. \\
\left.+\frac{1}{4} V^{-2} G^{i j}(b) a_{i} \wedge \star a_{j}+d \tilde{\lambda}^{i} \wedge a_{i}\right]-\sum_{n=0}^{N+1} \alpha_{i}^{(n)} \int_{M_{4}^{(n)}}\left(\tilde{\lambda}^{i}+\frac{b^{i}}{V} \sqrt{f^{*} g}\right) .
\end{array}
$$

Here $\lambda$ is a Lagrange multiplier, the $a_{i}$ arise from the Kaluza-Klein reduction of $G$ with respect to a basis of $H^{4}(\mathcal{M}), G_{i j}$ is the metric on the Kähler moduli space (not the 5dimensional metric which is denoted by $g_{M N} ; f^{*} g$ is its pullback to the three-brane worldvolume) and $\mathcal{K}$ its prepotential;

$$
G_{i j}=-\frac{1}{2} \frac{\partial^{2}}{\partial b_{i} \partial b_{j}} \ln \mathcal{K}, \quad \mathcal{K} \equiv \frac{1}{3 !} d_{i j k} b^{i} b^{j} b^{k},
$$

where $d_{i j k}$ are the intersection numbers of the $(1,1)$ forms on $\mathcal{M}$. The gravitational coupling $\ell$ is that of the original eleven-dimensional theory, and $L$ the length scale of the Calabi-Yau.

The boundary terms include the relevant contributions from $N$ five-branes at a priori arbitrary locations, with charges $\alpha_{i}^{(1)}, \ldots, \alpha_{i}^{(N)}$. In units of the five-brane tension/charge 
these correspond to the multiplicity or equivalently the winding number of five-branes around the various two-cycles. The first order action includes the Bianchi identity, modified in the presence of five-branes, as the field equation for the dual potential $\tilde{\lambda}^{i}$

$$
d a_{i} \equiv J_{i}^{(5)} \Rightarrow\left\{\begin{array}{c}
\partial_{11} a_{i}=\frac{2 \ell^{9}}{L^{6}} \sum_{k=0}^{N+1} \alpha_{i}^{(k)} \delta\left(x^{11}-x_{k}^{11}\right) \\
\partial_{\mu} a_{i}=0
\end{array}\right.
$$

with solution [5, 6]

$$
a_{i}=\frac{2 \ell^{9}}{L^{6}}\left(\sum_{k} \alpha_{i}^{(k)} \epsilon\left(x^{11}-x_{k}^{11}\right)+c_{i}\right) .
$$

The constants $\alpha_{i}^{(0)}, \alpha_{i}^{(N+1)}$ are the effective M5-brane charges carried by the end-of-theuniverse 9-plane domain-walls. On each end-of-the-universe plane, the effective five-brane charge has two constituents, similar to the effective $D p^{\prime}, \quad\left(p^{\prime}<p\right)$ charge carried by $D p$ branes in nontrivial backgrounds. One constituent is the $E_{8}$ instanton number, which may be interpreted as M5-branes immersed in the 9-brane [5, 24]; the second is the induced five-brane charge due to the non-vanishing curvature of the Calabi-Yau and proportional to $c_{2}(\mathcal{M})$ [5, 6]

$$
\alpha_{i}^{(k)}=\frac{T_{5} L^{2}}{8 \pi^{2}} \int_{D_{i}}\left(\operatorname{tr} F^{(k)} \wedge F^{(k)}-\frac{1}{2} \operatorname{tr} R \wedge R\right), \quad k=0, N+1 .
$$

Here $D_{i}$ is a 4 -cycle of the Calabi-Yau, and the elementary five-brane charge $T_{5}$ equals

$$
T_{5}=\frac{2 \pi}{(4 \pi)^{2 / 3} \ell^{6}} .
$$

In the remainder we set all scales to unity.

The total five-brane charge must vanish on the orbifold interval $S_{1} / \mathbb{Z}_{2}$;

$$
\sum_{k=1}^{N} \alpha_{i}^{(k)}+\sum_{j=0, N+1} \int_{D_{i}} \operatorname{tr} F^{(j)} \wedge F^{(j)}=\int_{D_{i}} c_{2}
$$

This is the Kaluza-Klein reduction of the invariant eleven-dimensional statement that the modified Bianchi identity [4]

$$
\begin{aligned}
d G= & \left(\left[\operatorname{tr} F_{(1)} \wedge F_{(1)}-\frac{1}{2} \operatorname{tr} R \wedge R\right] \delta(y)+\left[\operatorname{tr} F_{(2)} \wedge F_{(2)}-\frac{1}{2} \operatorname{tr} R \wedge R\right] \delta\left(y-\pi R_{11}\right)\right. \\
& \left.+\sum_{i} n_{5}^{i} \delta_{C^{i}} \delta\left(y-y_{i}\right)\right) \wedge d y
\end{aligned}
$$

due to the presence of boundaries and five-branes is integrable, i.e.,

$$
\int_{S_{1} / \mathbb{Z}_{2} \times D_{i}} d G=0 \quad \forall \quad D_{i} \in H_{4}(\mathcal{M}) .
$$


In (8) $\left\{C^{i}\right\}$ is a basis of $H_{2}(\mathcal{M})$ dual to the basis $\left\{D_{i}\right\}$ of $H_{4}(\mathcal{M})$ and $\delta_{C^{i}}$ is the fourform Poincaré dual to the 2-cycle $C^{i}$ with delta-function support on $C^{i}$. Preservation of supersymmetry allows only configurations of instantons and five-branes (or anti-instantons and anti-branes).

If one chooses the standard embedding of the spin connection in the first $E_{8}$ gauge group such that

$$
\operatorname{tr} F^{(1)} \wedge F^{(1)}=\operatorname{tr} R \wedge R
$$

then $\alpha_{i}^{(1)}=-\alpha_{i}^{(2)}$, no additional five-branes are needed and the effective five-brane charge on the Horava-Witten-plane with unbroken gauge group at $y=\pi R_{11}$ is just the topologically induced charge $\alpha_{i}^{(2)}=-\frac{1}{2} \int_{D_{i}} c_{2}$.

In this formulation the fields $a_{i}$ appear algebraically and may be integrated out, yielding the 4-dimensional domain-wall action with electric 5-form field-strengths $\mathcal{F}^{i}=d \tilde{\lambda}^{i}$;

$$
\begin{array}{r}
S_{5}=-\int_{M_{5}} \sqrt{-g}\left(R+G_{i j} \partial_{M} b^{i} \partial^{M} b^{j}+\frac{1}{2} V^{-2} \partial_{M} V \partial^{M} V+\lambda(\mathcal{K}-1)\right) \\
\left.-V^{2} G_{i j}(b) \mathcal{F}^{i} \wedge * \mathcal{F}^{j}\right]-\sum_{n=0}^{N+1} \alpha_{i}^{(n)} \int_{M_{4}^{(n)}}\left(\tilde{\lambda}^{i}+\frac{b^{i}}{V} \sqrt{f^{*} g}\right) .
\end{array}
$$

The supersymmetric domain-wall or three-brane solution to the field equations is given by [6]

$$
\begin{aligned}
d s_{5}^{2} & =e^{2 A} d x_{4}^{2}+e^{8 A} d y^{2} \\
V & =e^{6 A}, \\
e^{3 A} & =\left(\frac{1}{3 !} d_{i j k} f^{i} f^{j} f^{k}\right), \\
b^{i} & =e^{-A} f^{i}, \\
\mathcal{F}_{11, \mu \nu \rho \sigma}^{i} & =\epsilon_{\mu \nu \rho \sigma} e^{-10 A}\left(-\partial_{11} b^{i}+2 b^{i} \partial_{11} A\right),
\end{aligned}
$$

where the $f^{i}$ 's are defined in terms of one-dimensional harmonic functions

$$
\begin{aligned}
d_{i j k} f^{j} f^{k}=H_{i}, \quad H_{i} & =\sum_{n} \alpha_{i}^{(n)}\left|y-y_{n}\right|+c_{i} \\
& =\sum_{n=0}^{k} 2 \alpha_{i}^{(n)} y+k_{i}, \quad y_{k}<y<y_{k+1}
\end{aligned}
$$

and the $k_{i}$ are arbitrary constants of integration.

The solution can be obtained from the supersymmetry variations by searching for a global Killing spinor [6, 9, 23].

$$
\delta \psi_{\mu}^{A}=\gamma_{\mu}\left((\partial A) \gamma_{11} \epsilon^{A}-\frac{e^{4 A} b^{i} a_{i}}{6 V}\left(\tau_{3}\right)_{B}^{A} \epsilon^{B}\right),
$$




$$
\begin{aligned}
\delta \psi_{11}^{A} & =\partial \epsilon^{A}-\frac{e^{4 A} b^{i} a_{i}}{12}\left(\tau_{3}\right)_{B}^{A} \gamma_{11} \epsilon^{B}, \\
\delta \zeta^{A} & =(\partial \ln V) \gamma_{11} \epsilon^{A}-\frac{e^{4 A} b^{i} a_{i}}{V}\left(\tau_{3}\right)_{B}^{A} \epsilon^{B}, \\
\delta \lambda^{i A} & =\left(\partial b^{i}\right) \gamma_{11} \epsilon^{A}+\frac{e^{4 A}}{2 V}\left(a^{i}-\frac{2}{3} b^{j} a_{j} b^{i}\right)\left(\tau_{3}\right)_{B}^{A} \epsilon^{B} .
\end{aligned}
$$

The Killing spinor,

$$
\epsilon^{A}=e^{A}\left(\delta_{B}^{A}+\gamma_{11}\left(\tau_{3}\right)_{B}^{A}\right) \epsilon_{0}^{B},
$$

is preserved, provided the following conditions are satisfied

$$
\begin{aligned}
\partial A-\frac{e^{-2 A}}{6} b^{i} a_{i} & =0, \\
\partial b^{i}+\frac{e^{-2 A}}{2}\left(a^{i}-\frac{2}{3}(b \cdot a) b^{i}\right) & =0 .
\end{aligned}
$$

These are direct analogues of the attractor equations describing spherically symmetric BPS black-holes in four- and five-dimensional supergravities with eight supercharges [26, 27, 28]. By contracting the second equation in (17) with $\partial b^{j} G_{j i}(b)$, the third term vanishes whereas the first term yields the length (in the moduli space) of the vector tangent to the path the solution follows. Defining a central charge $Z=b^{i} a_{i}$, and using the fact that the $a_{i}=$

$\alpha_{i}^{(n)} \epsilon\left(y-y_{n}\right)$ are (piecewise) constant the resulting equation exhibits the monotonic flow of $Z$

$$
\partial Z=-2 e^{2 A} \partial b^{i} G_{i j} \partial b^{j} \leq 0 .
$$

Alternatively one can define the dimensionful central charge $\tilde{Z}=u^{i} \alpha_{i}$ where $u^{i}=e^{-2 A} b^{i}$. The attractor equation can be written in the form

$$
\partial \tilde{Z}=-\frac{1}{2} \partial_{i} \tilde{Z} G^{i j}(u) \partial_{j} \tilde{Z} \leq 0
$$

which relates the flow in the target space with the flow in the moduli space. Here,

$$
\partial_{i} \tilde{Z}=\frac{\partial \tilde{Z}}{\partial u^{i}}
$$

Notice that as in [6], we are holding the hypermultiplets (which control the complex structure) fixed, and we shall continue to do so throughout this paper. It would be interesting to relax this assumption as one would need to do to study, for example, conifold transitions.

\section{An Example}

An essential aspect of (12) is that the moduli of the Calabi-Yau manifold will generically vary along the transverse direction $y$. This raises the question of whether one can find solutions in which the Kähler moduli vary through a Kähler wall in the moduli space; i.e. the Calabi-Yau manifold undergoes a flop transition as we move along $y$. 
Suppose we are able to find such a solution; this will have consequences for the analysis of the action and the field equations in the previous section. Specifically, notice that the equations (12) and (13) depend upon the second Chern numbers through $\alpha_{i}^{(0)}$ and $\alpha_{i}^{(N+1)}$, and the intersection numbers $d_{i j k}$ of the Calabi-Yau. These numbers jump when a CalabiYau manifold undergoes a flop. Hence, to exhibit an example where topology change occurs, we must search for solutions that (a) pass through a wall of the Kähler moduli space and (b) are a solution to the field equations of the form (12) for values of the topological numbers $d_{i j k}$ and $c_{2}$ appropriate to the Calabi-Yau on each side of the wall. Furthermore, in order to trust the supergravity approximation in which we work, we must also ensure that the overall Calabi-Yau volume $V$ stays large.

In this section, through an explicit example involving some fairly tedious algebra, we will show that such topology changing solutions can indeed be found. In this example we will choose the standard embedding for the gauge bundles as this will facilitate our discussion of the Bianchi identity and flop-curves as sources of $G$-flux, in the next section.

A simple example of a pair of Calabi-Yau manifolds connected by a flop transition are the well studied $\left(h^{1,1}, h^{2,1}\right)=(3,243)$ Calabi-Yau manifolds considered in 17, 18, 19, 20, 21. To investigate whether a flop can occur dynamically, we will attempt to match the solutions to the field equations for each of the Calabi-Yau manifolds across the singular point. We will find that, though the values of the fields can be matched continuously, their first derivatives are discontinuous at the flop. The jumps in the first derivatives of the Kähler moduli imply that there is an additional source of magnetic under the $G$ flux charge where the solutions are matched together (the flop point), and the Kähler moduli are sensitive to this magnetic source. In the next section, we will discuss the generality of this class of solutions.

Before we proceed, let us collect the essential data of the Calabi-Yau and its flopped cousin [18]. Details can be found in the aforementioned papers. In these works the manifold

we denote with $\mathcal{M}$ is known as model III and $\widetilde{\mathcal{M}}$ as model II. Both are elliptic fibrations over Hirzebruch surfaces.

\section{Calabi-Yau Manifold $\mathcal{M}$}

The intersection numbers are summarized in the classical prepotential $\mathcal{K}_{\mathcal{M}}=\frac{1}{3 !} d_{i j k} t^{i} t^{j} t^{k}$. For $\mathcal{M}$ the prepotential equals:

$$
\mathcal{K}_{\mathcal{M}}=\frac{1}{3 !}\left(8\left(t^{1}\right)^{3}+9\left(t^{1}\right)^{2} t^{2}+3 t^{1}\left(t^{2}\right)^{2}+6\left(t^{1}\right)^{2} t^{3}+6 t^{1} t^{2} t^{3}\right)
$$

The $t^{i}$ are the components of the Kähler form expanded in a natural basis (a basis in which the Kähler cone is defined by $\left.t^{i}>0\right)$ of 2 -forms $\omega_{i}$ for $H^{2}(\mathcal{M}, \mathbb{Z})$,

$$
J=t^{i} \omega_{i}
$$

Integrating $J$ over an arbitrary 2-cycle $C^{i}$ shows that the corresponding $t^{i}$ measures its size.

The moduli $t^{i}$ are related to the dimensionless fields $b^{i}$ defined in the previous section by $b^{i}=V^{-1 / 3} t^{i}$. Therefore, the dependence of $t^{i}$ on the transverse direction $y$ is governed by the one-dimensional harmonic functions $H_{i}(y)$. The slopes of these harmonic functions are 
in turn determined by the effective five-brane charges. For the end of the world branes with standard embedding, the slopes are proportional to the periods of the second Chern class; in terms of the divisors $D_{i} \in H_{4}(\mathcal{M})$ dual to $\omega_{i}$, they are

$$
c_{2}\left(D_{1}\right)=92 \quad, \quad c_{2}\left(D_{2}\right)=36 \quad, \quad c_{2}\left(D_{3}\right)=24 \text {. }
$$

\section{Flopped Calabi-Yau Manifold $\widetilde{\mathcal{M}}$}

The intersection numbers of this Calabi-Yau may be determined from its cousin $\mathcal{M}$ with the help of the relation (see, e.g., [1])

$$
\left(D_{i_{1}} \cap D_{i_{2}} \cap D_{i_{3}}\right)_{\widetilde{\mathcal{M}}}=\left(D_{i_{1}} \cap D_{i_{2}} \cap D_{i_{3}}\right)_{\mathcal{M}}-\sum_{\beta} \prod_{k}\left(D_{i_{k}} \cap C^{\beta}\right)
$$

The $C^{\beta}$ represent the curves that are flopped and the $D_{i}$ on $\widetilde{\mathcal{M}}$ are the proper transforms of the $D_{i}$ on $\mathcal{M}$. In the present case there is only one such curve, and its intersection with the $D_{i}$ is $D_{i} \cap C=\delta_{i 3}$. Hence, the only intersection number which changes is $d_{333}$. It is shifted by -1 . Thus, if we choose the $D_{i}$ and their proper transforms as bases on $\mathcal{M}$ and $\widetilde{\mathcal{M}}$ we find that the prepotential of $\widetilde{\mathcal{M}}$ is equal to

$$
\mathcal{K}_{\widetilde{\mathcal{M}}}=\mathcal{K}_{\mathcal{M}}-\frac{\left(t^{3}\right)^{3}}{6}
$$

The Chern coefficients $\tilde{c}_{2}\left(D_{i}\right)$ of the flopped Calabi-Yau $\widetilde{\mathcal{M}}$ can be calculated from the Tian-Yau theorem in eq. (60) or using the relation

$$
\int_{D_{i}} c_{2}+2\left(D_{i} \cap D_{i} \cap D_{i}\right)=12 \chi\left(D_{i}\right)
$$

and noting that the holomorphic Euler characteristic $\chi\left(D_{i}\right)$ is invariant under flops. Hence,

$$
\tilde{c}_{2}\left(D_{1}\right)=92 \quad, \quad \tilde{c}_{2}\left(D_{2}\right)=36 \quad, \quad \tilde{c}_{2}\left(D_{3}\right)=26 .
$$

\section{Solutions to the field equations}

To find the explicit dependence of the moduli $f^{i}=V^{-1 / 6} t^{i}$ on the transverse direction we need to invert the nonlinear relations for $\mathcal{M}$ and $\widetilde{\mathcal{M}}$ between $f^{i}$ and $H_{i}$. As mentioned, we consider the case of standard embedding i.e., $\operatorname{tr} F \wedge F=\operatorname{tr} R \wedge R$ at $y=0$ and $\operatorname{tr} F \wedge F=0$ at $y=\pi R_{11}$. In that case the slopes $\alpha_{i}$ of the harmonic functions $H_{i}$ are solely proportional to periods of the second Chern class:

$$
\alpha_{i}=\frac{1}{16 \pi^{2}} \int_{D_{i}} \operatorname{tr} R \wedge R=\frac{1}{2} \int_{C Y} \omega_{i} \wedge c_{2}(C Y) .
$$


First we choose a more convenient basis [18, 20],

$$
\begin{aligned}
& t^{1}=U, \\
& t^{2}=T-\frac{1}{2} U-W, \\
& t^{3}=W-U .
\end{aligned}
$$

In this basis the respective Kähler cones of $\mathcal{M}$ and $\widetilde{\mathcal{M}}$ are defined by the regions

$$
\begin{array}{ll}
\mathcal{M}: & W>U>0, T>\frac{1}{2} U+W, \\
\widetilde{\mathcal{M}}: & U>W>0, T>\frac{3}{2} U .
\end{array}
$$

and the area of the flop curve equals $W-U$.

The relation between the moduli and the harmonic functions can now be straightforwardly inverted [21]. For $\mathcal{M}$ one finds

$$
\begin{aligned}
T & =\frac{1}{2} \frac{H_{T}}{U} \\
U & =\frac{1}{2} \sqrt{\left(H_{U}+\frac{1}{2} H_{W}\right) \pm \sqrt{\left(H_{U}+\frac{1}{2} H_{W}\right)^{2}+2 H_{W}^{2}-2 H_{T}^{2}}} \\
W & =-\frac{1}{2} \frac{H_{W}}{U}+\frac{1}{2} U
\end{aligned}
$$

with

$$
\begin{aligned}
H_{T} & =18|y|+k_{T}, \\
H_{U} & =25|y|+k_{U}, \\
H_{W} & =-6|y|+k_{W},
\end{aligned}
$$

and for $\widetilde{\mathcal{M}}$

$$
\begin{aligned}
T & =\sqrt{\frac{1}{2} \tilde{H}_{u} \pm \frac{1}{2} \sqrt{\tilde{H}_{u}^{2}-\frac{9}{4} \tilde{H}_{T}^{2}}}, \\
U & =\frac{2}{3} \sqrt{\frac{1}{2} \tilde{H}_{u} \mp \frac{1}{2} \sqrt{\tilde{H}_{u}^{2}-\frac{9}{4} \tilde{H}_{T}^{2}}} \\
W & =\sqrt{-\tilde{H}_{W}}
\end{aligned}
$$

with

$$
\begin{aligned}
\tilde{H}_{T} & =18|y|+\tilde{k}_{T}, \\
\tilde{H}_{U} & =24|y|+\tilde{k}_{U}, \\
\tilde{H}_{W} & =-5|y|+\tilde{k}_{W} .
\end{aligned}
$$

Requiring that we are in the correct Kähler cone for $\widetilde{\mathcal{M}}$ selects the positive and negative sign in the expressions for $T_{\widetilde{\mathcal{M}}}$ and $U_{\widetilde{\mathcal{M}}}$ respectively. 


\subsection{Matching the solutions}

Location of the flop

If the field equations (whose behaviour within the framework on each of the Calabi-Yau's we have already deduced) allow for a topology changing transition, it must be possible to match the solutions across the point where the flop-curve $t^{3}=W-U$ degenerates. As the relation between one of the moduli, $W$, of the Calabi-Yau $\widetilde{\mathcal{M}}$ and the harmonic functions is rather simple, we will first consider the flop solution from its point of view.

From the data in (33) and (34) we see that at the flop point the following quantity must vanish [21]

$$
9 \tilde{H}_{W}^{2}+4 \tilde{H}_{U} \tilde{H}_{W}+\tilde{H}_{T}^{2}=0 .
$$

Substituting the harmonic functions we find a quadratic equation for $y$. This equation has a solution if the discriminant is positive. The latter is a quadratic expression in the three integration constants $\tilde{k}_{i}$ and vanishes at the two roots

$$
25 \tilde{k}_{U}=\left(45 \pm \frac{5 \sqrt{69}}{2}\right) \tilde{k}_{T}+(42 \pm 9 \sqrt{69}) \tilde{k}_{W} .
$$

A brief inspection shows that large $\left|\tilde{k}_{U}\right|$ corresponds to a positive discriminant and for a flop to occur we must therefore tune our integration constants such that

$$
25 \tilde{k}_{U}>\left(45 \pm \frac{5 \sqrt{69}}{2}\right) \tilde{k}_{T}+(42 \pm 9 \sqrt{69}) \tilde{k}_{W}
$$

or

$$
25 \tilde{k}_{U}<\left(45 \pm \frac{5 \sqrt{69}}{2}\right) \tilde{k}_{T}+(42 \pm 9 \sqrt{69}) \tilde{k}_{W}
$$

Regardless of the flop a necessary condition is that at the location of the brane at $y=0$, we are using the correct Calabi-Yau data. One condition, $2 T>3 U$, is guaranteed by the choice of solution in (33). The second one, $W>0$, is obeyed by requiring $\tilde{k}_{W}<0$. The last constraint, $W<U$, yields a lower bound on $\tilde{k}_{U}$ in terms of $\tilde{k}_{T}$ and $\tilde{k}_{W}$

$$
\tilde{k}_{U}<-\frac{9 \tilde{k}_{W}^{2}+\tilde{k}_{T}^{2}}{4 \tilde{k}_{W}} .
$$

Thirdly, by requiring that (33) has solutions, we see that $\tilde{H}_{U}^{2}>9 \tilde{H}_{T}^{2} / 4$ or $2\left|\tilde{k}_{U}\right|>3\left|\tilde{k}_{T}\right|$. Fourthly, in order that the solution to (35) truly corresponds to a point where $U=W$, the lower bound

$$
\tilde{k}_{U}>\frac{\tilde{k}_{W}}{2}
$$

must be satisfied. Finally, in order that all divisors also have positive area at $y=0$ one finds an additional constraint $\tilde{k}_{T}>0$.

The parameter space that obeys all these conditions consists of two regions, and it is quite generic that a flop occurs for some choice of intitial conditions. The two regions correspond to 


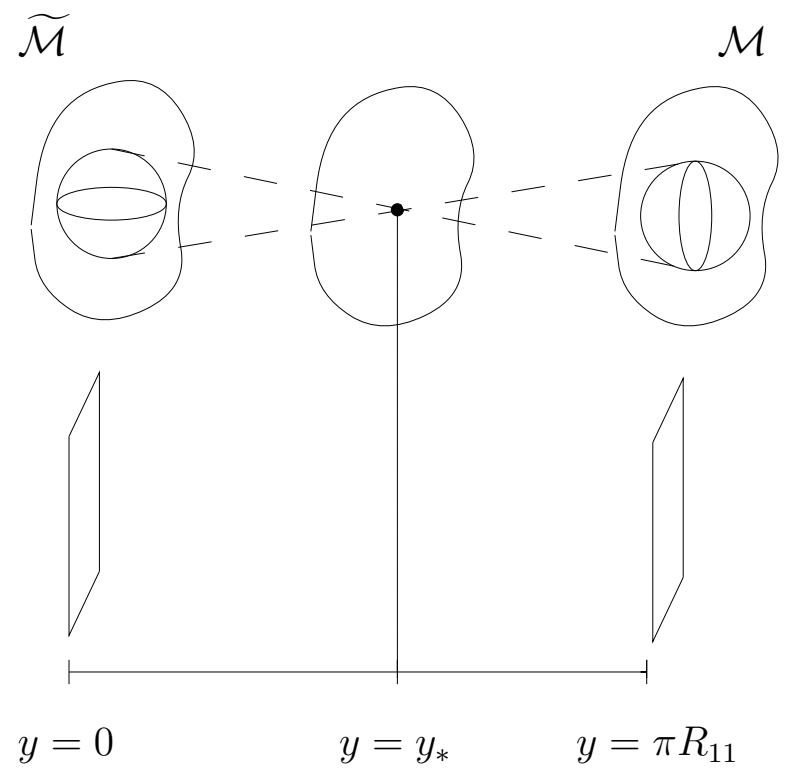

Figure 1: Calabi-Yau configuration in heterotic M theory which undergoes a flop.

two possible flop scenarios: (a) both roots, $y_{+}$and $y_{-}$, of (35) are larger than zero or (b) both roots are less than zero. A priori, there is the third possibility that $y_{+}>0>y_{-}$. However, this is not allowed because the quadratic (35) is convex and were we to find $y_{+}>0>y_{-}$, $W$ would be larger than $U$ at the location of the brane at $y=0$. Hence we would be in the wrong Kähler cone.

Given the explicit values of the roots,

$$
\begin{aligned}
y_{ \pm} & =\frac{1}{69}\left(10 \tilde{k}_{U}-18 \tilde{k}_{T}-3 \tilde{k}_{W} \pm \sqrt{\mathcal{D}}\right), \\
\mathcal{D} & =-612 \tilde{k}_{W}^{2}-336 \tilde{k}_{U} \tilde{k}_{W}+108 \tilde{k}_{T} \tilde{k}_{W}+100 \tilde{k}_{U}^{2}-360 \tilde{k}_{U} \tilde{k}_{T}+255 \tilde{k}_{T}^{2},
\end{aligned}
$$

the location of the minimum of (35) is

$$
y_{\text {min }}=\frac{1}{69}\left(10 \tilde{k}_{U}-18 \tilde{k}_{T}-3 \tilde{k}_{W}\right) .
$$

We will consider the region of parameter space such that $y_{\min }$ is positive.'] It is easy to see that $y_{\min }>0$ for large $\tilde{k}_{U}$ provided that the other $\tilde{k}_{i}$ obey the above constraints (for example, $k_{U}=100, k_{T}=30$ and $k_{W}=1$ ). This region thus corresponds to scenario (a); the Calabi-Yau manifold at $y=0$ is model II with an $S U(3)$ gauge bundle and that at $y=\pi R$ is model III with no gauge bundle.

\section{Continuity}

We demand that the solution for the 2-cycles $b^{i}=V^{-1 / 6} f^{i}$ can be matched across the flop at the flop location $y_{*} \equiv y_{-}$. This implicitly requires that the harmonic functions match

\footnotetext{
${ }^{1}$ As it turns out, the field equations do not allow topology changing solutions with standard embedding in scenario (b).
} 
as well; $H_{i}\left(y_{*}\right)=\tilde{H}_{i}\left(y_{*}\right)$. As $c_{2}\left(D_{T}\right)=\tilde{c}_{2}\left(D_{T}\right)$ this implies that $\tilde{H}_{T}(y)=H_{T}(y)$ for all $y$. Similarly $H_{U}(y)+H_{W}(y)=\tilde{H}_{U}(y)+\tilde{H}_{W}(y)$. Combining this with the final requirement that $H_{U}\left(y_{*}\right)-H_{W}\left(y_{*}\right)=\tilde{H}_{U}\left(y_{*}\right)-\tilde{H}_{W}\left(y_{*}\right)$ yields

$$
\begin{aligned}
k_{T} & =\tilde{k}_{T} \\
k_{U}+k_{W} & =\tilde{k}_{U}+\tilde{k}_{W} \\
k_{U}-k_{W} & =\tilde{k}_{U}-\tilde{k}_{W}-\frac{1}{2} y_{*}\left(\left(c_{2}(U)-\tilde{c}_{2}(U)\right)-\left(c_{2}(W)-\tilde{c}_{2}(W)\right)\right) .
\end{aligned}
$$

In order that $U_{\mathcal{M}}\left(y_{*}\right)-W_{\mathcal{M}}\left(y_{*}\right)$ indeed vanishes at the flop point on Calabi-Yau $\mathcal{M}$ with the above values of $k_{i}$, we need to choose the negative sign in front of the square root in (31).

It is now straightforward to plot the solutions on both side of the flop for a particular choice of constants $k_{i}$ in region (a) and establish the occurence of a flop transition; see figure 2.

\section{Discontinuity in the first derivatives}

We have seen that the function values of the moduli in the Calabi-Yau manifold $\mathcal{M}$ can be continuously connected to that in the flopped Calabi-Yau $\widetilde{\mathcal{M}}$ at the location of the flop. We now check whether the first derivatives are continuous.

In a universal, basis independent, notation the derivatives of the 2-cycles $f^{i}$ are related to those of the harmonic functions as

$$
M_{i j} \partial f^{j}=\partial H_{i}
$$

Here $M$ is the symmetric matrix $M_{i j}=2 d_{i j k} f^{j}$. This equation is nothing but the condition for the existence of a global Killing spinor (17) and therefore a component version of the attractor equation (19). Indeed $M_{i j} \partial f^{j} \sim G_{i j} \partial b^{j}$.

In a natural orthogonal basis such as the $t^{i}$ where exactly one of the $t^{i}$ shrinks to zero, one sees immediately that at the flop point $M$ is continuous. The potential source of discontinuity, the jump in the intersection numbers, always multiplies the very cycle which goes to zero. Recalling that the derivative of the harmonic function is just the slope,

$$
\partial f^{j}=\left(M^{-1}\right)^{j i} \alpha_{i}
$$

continuity of the first derivative of the cycles rests solely on the continuity of the slopes. Here, knowing its relation to the moduli space metric, we assume that the matrix $M$ is invertible. The slopes, of course, are the five-brane charges at each end of the universe. And these jump due to the flop transition:

$$
\begin{aligned}
\partial f^{j}\left(y_{*}\right)-\partial \tilde{f}^{j}\left(y_{*}\right) & =\left(M^{-1}\left(y_{*}\right)\right)^{j i} \alpha_{i}-\left(\tilde{M}\left(y_{*}\right)^{-1}\right)^{j i} \tilde{\alpha}_{i} \\
& =\left(M^{-1}\left(y_{*}\right)\right)^{j i}\left(\alpha_{i}-\tilde{\alpha}_{i}\right)
\end{aligned}
$$

Since $M$ is generically not (block-)diagonal, the first derivatives of all cycles are discontin-

uous, as is evident in figure 2. The reason for the jump is the mismatch in $\int_{D_{i}} c_{2}(R)$ on different sides of the flop. 


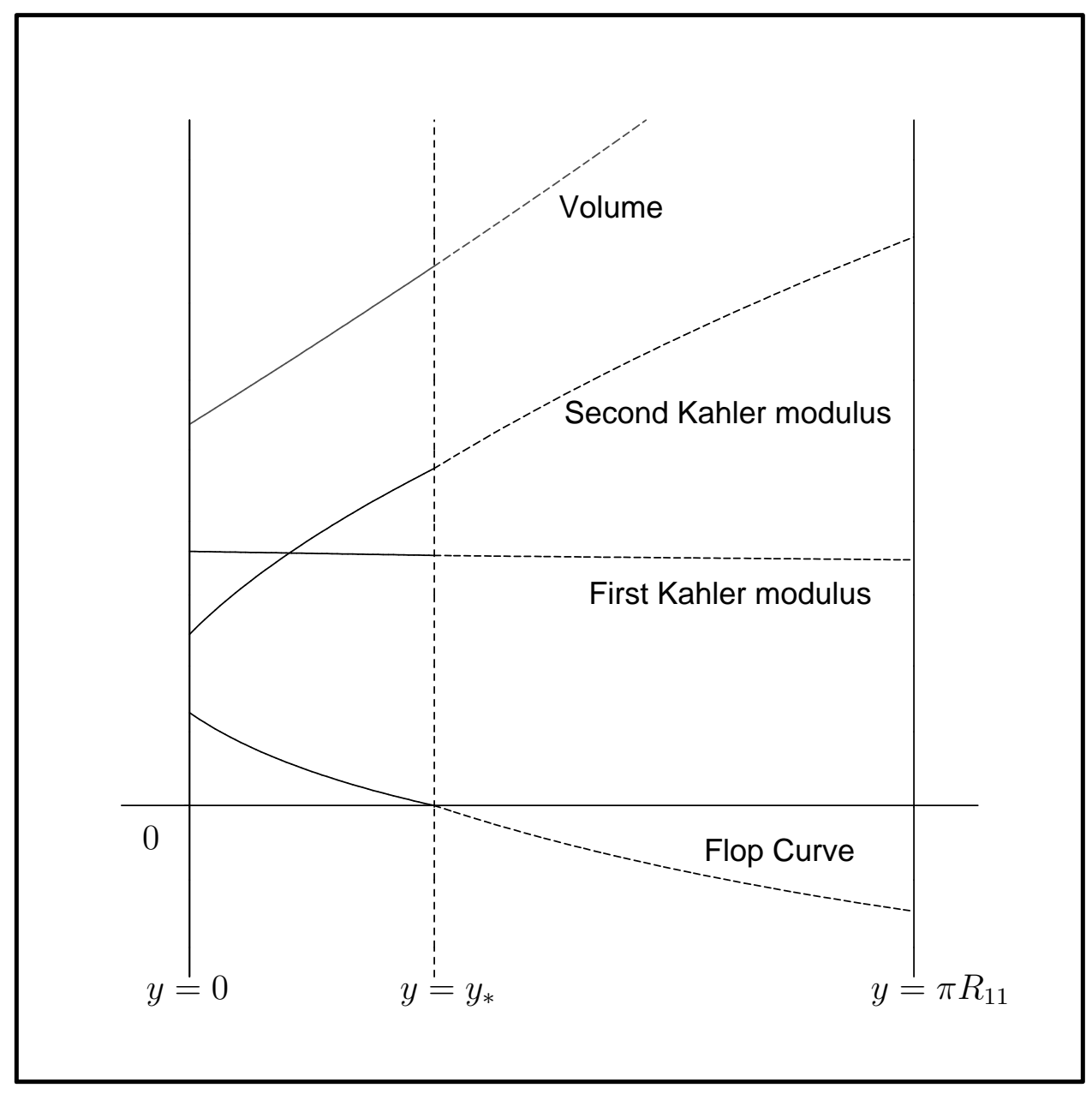

Figure 2: Profile of the areas of the two cycles and the Calabi-Yau volume along $y$ in a basis appropriate for Calabi-Yau $\widetilde{\mathcal{M}}$. Solid lines belong to the validity region $y<y_{*}$ of the Calabi-Yau $\widetilde{\mathcal{M}}$. Dashed lines belong to the Calabi-Yau $\mathcal{M}$ and hold for $y>y_{*}$.

Let us also briefly check the behaviour of the volume $V$ of the Calabi-Yau,

$$
V=\left(\frac{1}{3 !} d_{i j k} f^{i} f^{j} f^{k}\right)^{2}=\frac{1}{36}\left(f^{i} H_{i}\right)^{2} .
$$

It is obviously continuous for the same reason that $M$ is continuous. As for its derivative, it equals

$$
\partial V=\frac{\sqrt{V}}{3}\left(\partial f^{i} H_{i}+f^{i} \partial H_{i}\right)
$$

Using the above result for $\partial f^{i}$ and the implicit relation $f^{i}=\frac{1}{2} M^{-1}(f(H)) H$, this yields

$$
6 \partial \sqrt{V}=\left(\partial H \cdot M^{-1} \cdot H+\frac{1}{2} H \cdot M^{-1} \cdot \partial H\right) .
$$


and one recognizes the first Killing spinor equation in (17). Again the expression is simplified at the flop; and one would guess that the volume is discontinuous as well, as $H_{i}(y)$ and $M^{-1}(y)$ are continuous, but the slopes $\partial H_{i}(y)$ are not

$$
\partial \sqrt{V}-\partial \sqrt{\tilde{V}}=\frac{1}{4}\left((\alpha-\tilde{\alpha}) \cdot M^{-1} \cdot H\right) .
$$

However, using the implicit relation, $f^{i}=\frac{1}{2} M^{-1} H$, once more, this is seen to be equal to

$$
\partial \sqrt{V}-\partial \sqrt{\tilde{V}}=\frac{1}{2}((\alpha-\tilde{\alpha}) \cdot f) .
$$

The only elements of the charge vector which jump are exactly those parallel to the flopping curve $f_{\text {flop }}^{i}$, which shrinks to zero. The potential source of discontinuity is therefore absent and $\partial V$ is in fact continuous. This also means that the second derivative of $V$ has no delta-function singularity.

\subsection{Physical properties of the flop point}

Charge of the flop

In comparing the solutions of the two topologically distinct Calabi-Yau manifolds at the flop, we find that the first derivatives of the Kähler moduli, $\partial_{11} b^{i} \sim \partial f^{i}$, are discontinuous.

By virtue of our gluing together solutions whose (piecewise) constant $G$ fluxes differ in magnitude, there is a jump in $G$ flux on crossing the flop point, and such a jump will cause a discontinuity in the fields. As we will discuss in more detail in the next section, this is part of why we will associate a magnetic $G$-charge with the flop point. More generally, though, for the $G$ flux to be globally defined, we require that

$$
\int d G=0
$$

which implies that the sum of magnetic charges under the four-form $G$ must vanish. If the topology of the Calabi-Yau does not change, this condition is manifest for the standard embedding configuration; the geometrical five-brane charge induced from $\operatorname{tr} R \wedge R$ is cancelled by that of the instanton configuration. However, as the topology of the Calabi-Yau on one "end of the world" changes, the global charge conservation constraint is no longer satisfied.

Denoting the induced magnetic charges, $\int_{D_{i}} G$, associated with the divisors $D_{i}$ of the Calabi-Yau manifolds by $\left(q_{1}, q_{2}, q_{3}\right)$, the brane at $y=0$ reduced over the flopped Calabi-Yau $\widetilde{\mathcal{M}}$ has induced geometric charge $\alpha_{\text {geom }}^{y=0}=-\frac{1}{2} \tilde{c}_{2}\left(D_{1}, D_{2}, D_{3}\right)=(-46,-18,-13)$ compensated by the instantons with charge $\alpha_{i n s t}^{y=0} \equiv \tilde{c}_{2}\left(D_{i}\right)=(92,36,26)$. The brane at $y=\pi R_{11}$ only has induced geometric charges which equal $\alpha_{\text {geom }}^{y=\pi}=-\frac{1}{2} c_{2}\left(D_{i}\right)=(-46,-18,-12)$. Adding all contributions we see that we have an excess of $q_{\text {total }}=(0,0,1)$ units of charge.

The question is: what accounts for the missing $(0,0,-1)$ units of magnetic charge? As indicated, a natural candidate is the flop curve which degenerates to zero volume at the flop point. This situation is not completely unfamiliar. There are examples where geometric 
singularities are magnetic sources (e.g. orbifold singularities in [16]). The singular object in the present setting is the degenerate flop curve. It is therefore natural to conjecture that the flop must in some way be accompanied by $(0,0,-1)$ units of charge to ensure global charge conservation. If this is so, the discontinuities in the solution to the field equations is just a consequence of the fact that the flop carries magnetic charges, thereby inducing a jump in the $G$-flux.

We will examine in more detail the nature of the charge at the flop point in the next section.

\section{Tension of the flop}

If the flop point is a charge source, it is natural to inquire as to its possibly being a stressenergy source as well. The tension may be deduced from the curvature singularity. For a metric of the form (12), the non-vanishing components of the Einstein tensor are

$$
\begin{aligned}
G_{\mu \nu} & =-3 e^{-6 A}\left(A^{\prime \prime}-2\left(A^{\prime}\right)^{2}\right) \eta_{\mu \nu}, \\
G_{11,11} & =-6 A^{\prime 2} .
\end{aligned}
$$

The delta function singularities of the Einstein tensor, indicating a source of tension, can only come from the term $A^{\prime \prime}$, where $e^{A}=V^{1 / 6}$. In the previous subsection we showed that the second derivative of $V$ is at most discontinuous. Hence, the tension of the flop point is zero. We note that this is in accord with the tension one would have from a five-brane wrapped around the flopping curve at the flop point.

\section{Supersymmetry}

Finally, we should check whether the charge of the flop is consistent with supersymmetry. In order that the supersymmetries which are preserved all have the same chirality, the inner products $f^{i} \alpha_{i}^{(n)}$ for all charged objects with charges $\alpha_{i}^{(n)}$ must have the same sign (from the Killing spinor equations, Eqs. (15))

$$
f^{i} \alpha_{i}^{(n)} \geq 0
$$

This inner product is equivalent to $\int_{C Y} J \wedge c_{2}(V)$ for vector bundles and $\int_{C^{(n)}} J$ for five branes wrapping a curve $C^{(n)}$. This gives the well known condition that in order to preserve supersymmetry the bundles must be holomorphic and stable, and that five-branes must wrap around holomorphic curves. See also [23] for an in depth analysis of the supersymmetry structure.

What about the proposed charge located at the flop curve? As the volume of a flopped curve $\int_{C} J$ vanishes at a flop point it preserves the global Killing spinor.

Note that since $f^{i}$ changes sign for the curve which flops, we see from eq. (54) that a supersymmetric five-brane wrapping around the flopping holomorphic curve on either side of the flop will have opposite charge $\alpha_{i}$. 2 This can be understood by noting that for a fixed

\footnotetext{
${ }^{2}$ Naively, one might think that it is possible to introduce as many 5-branes wrapping around the flopping curve $C$ as possible, provided that we introduce an equal number of 5 -branes wrapping around the curve $\tilde{C}$
} 
set of divisors $D_{j}$, if the flopping holomorphic curve $C$ meets, say, $D_{i}$ transversely with $C \cap D_{i}=+1$ in Calabi-Yau manifold $\mathcal{M}$, then the corresponding holomorphic curve $\tilde{C}$ on the flopped Calabi-Yau manifold $\widetilde{\mathcal{M}}$ lies inside $D_{i}$, with $\widetilde{C} \cap D_{i}=-1$.

\section{Topology Changing Solutions}

In the above example, we have seen that consistency of the solution suggests that the zerosize flop curve is a source of magnetic charge. The effect of a magnetic charge at the flop is to modify the Horava-Witten Bianchi identity so that $G$ is globally defined. In this section, we discuss this idea in more detail.

Let us begin by considering the global charge constraint anew. For simplicity, let us temporarily ignore the possibility of additional five-branes wrapped on other, non-degenerating, two-cycles in the bulk. In the eleven-dimensional theory on $S_{1} / \mathbb{Z}_{2}$, the Bianchi identity for

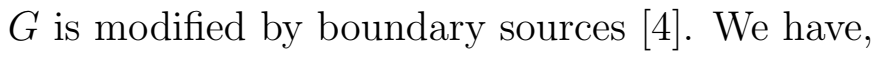

$$
\begin{aligned}
d G= & \left(\left[\operatorname{tr} F_{(1)} \wedge F_{(1)}-\frac{1}{2} \operatorname{tr} R_{(1)} \wedge R_{(1)}\right] \delta(y)\right. \\
& \left.+\left[\operatorname{tr} F_{(2)} \wedge F_{(2)}-\frac{1}{2} \operatorname{tr} R_{(2)} \wedge R_{(2)}\right] \delta\left(y-\pi R_{11}\right)\right) \wedge d y
\end{aligned}
$$

where we are now taking care to distinguish the curvatures of the different Calabi-Yau spaces at each end of the interval. In the usual case, where $\operatorname{tr} R_{(1)} \wedge R_{(1)}=\operatorname{tr} R_{(2)} \wedge R_{(2)}$, we have the familiar standard embedding solution

$$
\operatorname{tr} F_{(1)} \wedge F_{(1)}=\operatorname{tr} R \wedge R, \operatorname{tr} F_{(2)} \wedge F_{(2)}=0
$$

to the global consistency constraint that

$$
\int_{S_{1} / \mathbb{Z}_{2} \times D} d G=\int_{S_{1} / \mathbb{Z}_{2} \times D} d y\left(\frac{1}{2} \operatorname{tr} R \wedge R \delta(y)-\frac{1}{2} \operatorname{tr} R \wedge R \delta\left(y-\pi R_{11}\right)\right)=0 .
$$

But if $\operatorname{tr} R_{(1)} \wedge R_{(1)} \neq \operatorname{tr} R_{(2)} \wedge R_{(2)}$ (cohomologically) then the mismatch implies that solely embedding either spin connection into the gauge group is no longer a solution.

A natural suggestion, then, is to seek out different holomorphic stable bundles to place at $y=0$ and $y=\pi R_{11}$ with different second Chern classes, so as to find new consistent solutions to the Bianchi identity. Indeed, one may be able to find appropriate bundles to restore anomaly freedom. However, for a number of reasons, we propose a more general and universal solution. Namely, when $\operatorname{tr} R_{(1)} \wedge R_{(1)} \neq \operatorname{tr} R_{(2)} \wedge R_{(2)}$ because the Calabi-Yau has flopped somewhere along $y$, there is a new contribution to the Bianchi identity associated with the collapsed flop curves.

To motivate our proposal, notice that given a potential flop solution to (12), specifically with standard embedding, we can vary the location in $y$ at which the flop occurs, by varying

on the other side of the flop. However, one has to check that the resulting configuration still has a consistent topology changing solution to the field equations. 
the integration constants $k_{i}$ - as we have seen explicitly in the previous section. Imagine now that we have such a solution where a cycle shrinks to zero size but we choose the $k_{i}$ such that the flop does not occur in the physical range $0 \leq y \leq \pi R_{11}$; rather, it happens formally at $y=\pi R_{11}+\epsilon$. This is just the usual situation in which the topologies of the Calabi-Yau manifolds at $y=0$ and $y=\pi R_{11}$ are identical. Therefore, as expected, the choice of a standard embedding of the spin connection into the gauge group is consistent.

Let's now adjust the $k_{i}$ so that the flop occurs at $\pi R_{11}-\epsilon$, in the physical range so that the pure standard embedding, with no other charge sources, no longer provides a consistent solution. Where can the necessary other charge sources be? Four natural possibilities are: (1) Additional gauge structure at $y=0,(2)$ New gauge structure at $y=\pi R_{11}$, (3) New wrapped 5-branes, or (4) Magnetic charge associated with the geometrical singularity at $y_{*}$. By locality we do not expect to fix the Bianchi identity by adjusting the gauge bundle at $y=0$, as that lies at "the other end of the universe" from where the flop occurs, making possibility (1) seem unlikely. Nor do we expect to excite the new nontrivial gauge structure by changing the geometry/topology of the Calabi-Yau at $y=\pi R_{11}$ (where the initial gauge bundle was chosen to be trivial). In fact, as we will see in a moment, possibility (2) seems to be ruled out because the sign of the new magnetic source required to fix the Bianchi identity conflicts with the requirement that the new gauge bundle at $y=\pi R_{11}$ is stable. Possibility (3) immediately raises the question: where along $y_{11}$ should the purported new 5 -brane wrapping $C$ be located? From the discussion of the preceeding section, to contribute the correct charge, it must be located at a point $y$ with $y \leq y_{*}$. Locality, and consistency with the solutions we have constructed in section 3 in which the flux jumps at the flop point, pick out $y=y_{*}$. We will come back to this in a moment.

To understand possibility (4) we note that since a flop causes the geometrical $G$-source contribution $\operatorname{tr} R \wedge R$ to change, it is natural to suggest that a compensating magnetic source is provided by the geometrical singularity at the location of the flop itself. Strictly speaking, we are considering $\mathrm{M}$ theory compactified on a 7-manifold with boundaries, as the CalabiYau moduli are varying over the $S^{1} / \mathbb{Z}_{2}$. At the location of the flop, the 7 -manifold is also singular - with the singularity of the form of a cone over $C P^{3}$ (see, e.g., 25]) [. Geometrical singularities are known to carry magnetic charges (e.g. orbifold singularities in [16], and orientifolds). The singular objects in the present setting are the flop curves when they attain zero quantum volume.

To complete the proposal, we need to specify the strength of the charge carried by the flop. A theorem of Tian and Yau, which we will discuss directly below, suggests that, if a flop occurs at $y=y_{*}$, the gravitational contribution to the Bianchi identity is modified to

$$
d G_{\text {grav }}=\left(-\frac{1}{2} \operatorname{tr} R_{(1)} \wedge R_{(1)} \delta(y)-\frac{1}{2} \operatorname{tr} R_{(2)} \wedge R_{(2)} \delta\left(y-\pi R_{11}\right)+\sum_{\beta} \delta_{C^{\beta}} \delta\left(y-y_{*}\right)\right) \wedge d y,
$$

where $\beta$ sums over all the holomorphic curves $\left\{C^{\beta}\right\}$ that flop at $y=y_{*}$.

This additional gravitional contribution has a close analogy with bulk five-brane sources wrapped on nonsingular curves [16]. The gauge bundle contribution to $d G$ can be augmented

\footnotetext{
${ }^{3}$ We thank Edward Witten and Chien-Hao Liu for discussions on this point.
} 
by having five-branes wrapped on two-cycles $C^{i} \subset \mathcal{M}$ at locations $y=y_{i}$. This causes the matter part of the Bianchi identity to take the form [5] (see eq. (8))

$$
d G_{\text {matter }}=\left(\operatorname{tr} F_{(1)} \wedge F_{(1)} \delta(y)+\operatorname{tr} F_{(2)} \wedge F_{(2)} \delta\left(y-\pi R_{11}\right)+\sum_{i} \delta_{C^{i}} \delta\left(y-y_{i}\right)\right) \wedge d y
$$

in the case of singly wrapped five-branes on holomorphic curves.

There is, however, an important subtlety in the above expression (58). In the presence of a flop, one has to define precisely what one means by the curves $C^{\beta}$. For with respect to a chosen basis of divisors $D_{i}$, which (via invoking the proper transform map) can be made universal on both sides of the flop, flopping curves differ in orientation from one side to the other. To be concrete, suppose that the flopping curve $C^{\beta}$ and the set of divisors $D_{i}$ meet transversely on a Calabi-Yau manifold $\mathcal{M}$ with $C^{\beta} \cap D_{i}=1$. In the flopped Calabi-Yau manifold $\widetilde{\mathcal{M}}$, as we mentioned earlier, the flopping curve $\widetilde{C}^{\beta}$ is no longer transverse to $D_{i}$, but instead lies inside $D_{i}$, with $\widetilde{C}^{\beta} \cap D_{i}=-1$. Therefore, one has to specify in Eq. (58) on which Calabi-Yau manifold the holomorphic curve $C^{\beta}$ is collapsing in order that the geometrical data is complete. In fact, this subtlety in assigning magnetic charges when a curve collapses is not intrinsic to the flop point, and is already present when one considers a five-brane wrapped around a collapsing curve. We have discussed this subtlety in the analysis of supersymmetry in the previous section. In the same manner, one has to specify on which Calabi-Yau manifold the curve $C_{i}$ (that the fivebrane wraps) is collapsing. The reason is that with respect to a fixed divisor $D_{i}$ (and its proper transform), the charge of the five-brane differs by a sign depending on whether one approaches the flop point from one Calabi-Yau or from its flopped cousin.

Let us examine more closely which holomorphic curve $C^{\beta}$ one should use in Eq. (58). Recall that our topology changing solution can be obtained, starting from a standard solution in which the topology of the Calabi-Yau does not change in the physical regime $0 \leq y \leq \pi R_{11}$ (which we call the "parent" solution). Subsequently by varying the initial conditions of the two-cycles (the integration constants $k_{i}$ ), while keeping the gauge bundle fixed, we can bring the flop point to $y=\pi R_{11}-\epsilon$. This causes a jump in $\operatorname{tr} R \wedge R$, which must be compensated by the flop charge. The question, then, is: What is the jump in $\operatorname{tr} R \wedge R$ under a flop? A theorem of Tian and Yau [15] states that starting from a Calabi-Yau manifold $\widetilde{\mathcal{M}}$ and a collection of holomorphic curves $\left\{C^{\beta}\right\}$ on $\widetilde{\mathcal{M}}$, the second Chern numbers of $\widetilde{M}$ and its flopped cousin $M$ are related by

$$
c_{2}(\mathcal{M})=c_{2}(\widetilde{\mathcal{M}})+2 \sum_{\beta} \int_{D}\left[C^{\beta}\right]
$$

with $D$ an arbitrary divisor and $\left[C^{\beta}\right] \epsilon H^{4}(\widetilde{\mathcal{M}})$, the Poincare dual of $C^{\beta}$. We therefore see that

$$
-\frac{1}{2} \operatorname{tr}_{\mathcal{M}} R_{(2)} \wedge R_{(2)}+\sum_{\beta} \delta_{C_{(1)}^{\beta}}=-\frac{1}{2} \operatorname{tr}_{\widetilde{\mathcal{M}}} R_{(1)} \wedge R_{(1)}
$$


Hence, the $C^{\beta}$ in (58) are holomorphic curves on the Calabi-Yau manifold $\widetilde{\mathcal{M}}$ of the "parent" solution. The total $G$ charge, including that carried by the flop, still equals $\operatorname{tr} R_{(1)} \wedge R_{(1)}$, which is exactly cancelled by the standard embedding on the brane wrapping $\widetilde{\mathcal{M}}$; the charge conservation constraint is satisfied.

Notice that the flop contribution to the Bianchi identity is the same as that of a fivebrane wrapping the flopping curve on the Calabi-Yau of the "parent" solution (which lies at $y=0$ in our setup). However due to the fact that supersymmetric five-branes wrapping the "flopping" curve on the other "flopped" Calabi-Yau, must carry the opposite charge, the missing charge required to satisfy the Bianchi identity cannot be carried by a five-brane on the other side of the flop. Nevertheless, this means that the magnetic source contributed by a wrapped 5-brane over the appropriate degeneration of the flop curve is identical to the magnetic source of the singularity at $y_{*}$ to which we are led by the result of Tian and Yau. Hence, at our level of analysis possibility (3) — realized at $y_{*}$ - and possibility (4) are indistinguishablet.

We can now also complete the promised argument that seems to rule out possibility (2). The missing magnetic source for $d G$ in the presence of a single flopped curve is $\delta_{C}=-\delta_{C^{\prime}}$ where $C$ lies on the Calabi-Yau to the left of $y_{*}$ and $C^{\prime}$ lies on the Calabi-Yau to the right of $y_{*}$. The question then is whether there is a stable holomorphic vector bundle $V$ on the Calabi-Yau at $y=\pi R_{11}$ such that

$$
c_{2}(V)=\frac{1}{2}\left(c_{2}\left(T_{2}\right)-c_{2}\left(T_{1}\right)\right)=-\delta_{C^{\prime}} .
$$

But if there were such a bundle, then

$$
\int c_{2}(V) \wedge J=-\int_{C^{\prime}} J<0
$$

violating the stability condition on $V$. Hence, a gauge instanton at $y=\pi R_{11}$ alone cannot account either for the missing charge required to satisfy the Bianchi identity.

We note that the inability of $G$-charge considerations to distinguish between a five-brane of the "parent" solution wrapping the zero-size flop curve and a purely geometrical singularity raises the interesting question whether flop transitions aside from new sources of five-brane charge are also accompanied by the introduction of new low-energy degrees of freedom

We propose therefore, that in strongly coupled heterotic string theory, the general Bianchi identity for arbitrary gauge bundles at $y=0$ and $y=\pi R_{11}$, in a spacetime background in which the Calabi-Yau undergoes a flop transition at $y=y_{*}$, together with an arbitrary

\footnotetext{
${ }^{4} \mathrm{An}$ argument for possibility (3) is, perhaps, that the above calculation indicates that the form of the local singularity does not uniquely determine the sign of the charge it is required to carry. Namely, as we have phrased the calculation, the additional data of which Calabi-Yau is used in the "parent" theory is needed. Equivalently, the sign of the charge depends upon the details of the background $G$-flux.

${ }^{5}$ We note that preliminary analysis indicates that we can deform the solutions of section III so that the jump in the $G$-flux occurs to the left of the flop point, with all quantitites then being smooth across the flop point itself. This would naturally be interpreted as the required $G$-charge being carried by a 5 -brane wrapped on the "would-be" flop curve, to the left of the flop point. Further analysis of these solutions would likely help clarify this issue.
} 
assortment of 5-branes wrapping two-cycles other than those involved in the flop transition itself, is

$$
\begin{aligned}
d G= & \left(\left[\operatorname{tr} F_{(1)} \wedge F_{(1)}-\frac{1}{2} \operatorname{tr} R_{(1)} \wedge R_{(1)}\right] \delta(y)+\left[\operatorname{tr} F_{(2)} \wedge F_{(2)}-\frac{1}{2} \operatorname{tr} R_{(2)} \wedge R_{(2)}\right] \delta\left(y-\pi R_{11}\right)\right. \\
& \left.+\sum_{i} n_{5}^{i} \delta_{C^{i}} \delta\left(y-y_{i}\right)+\delta\left(y-y_{*}\right) \sum_{\beta} \delta_{C^{\beta}}\right) \wedge d y .
\end{aligned}
$$

with $C^{i}$ being holomorphic curves in the Calabi-Yau that the corresponding five-brane wraps, and $C^{\beta}$ being the flop curve on the appropriate Calabi-Yau as described above. Of course, the last two contributions can be grouped together, so long as the contribution associated with any degenerating curves is calculated according to the limiting procedure from the correct side of the flop point.

\section{Conclusion}

In this paper, we have studied topology changing solutions in M theory compactifications on Calabi-Yau three-folds with nonzero $G$ flux. In the presence of $G$ flux, the field equations do not admit five dimensional Minkowski space as vacuum solution. The spacetime metric is warped, with the solution of the domain-wall type so that one of the five dimensions is singled out as the transverse direction. In addition to the spacetime metric, other moduli of the Calabi-Yau manifold also vary along the transverse directions. We have studied an example in strongly coupled heterotic string theory - the supersymmetric domain wall solution in which the field equations force us to go through a flop transition as we move from one end of the world to the other. Consistency of the solution suggests that a flop curve - like an ordinary (wrapped) M theory fivebrane - carries a single unit of magnetic charge under the $G$ flux.

We have focussed our attention on flop transitions. As in [6] we have assumed that the hypermultiplets can be consistently decoupled in the five-dimensional effective theory. Especially in non-trivial geometric situations as presented here, it would be interesting to revisit this issue of decoupling. Moreover, this would allow the study of more drastic change of topology, such as conifold transitions, in which the hypermultiplets necessarily play a role. As the number of moduli of the Calabi-Yau changes across a conifold transition, it is perhaps more appropriate to treat the compact manifold as a $G_{2} 7$-manifold with boundaries which are Calabi-Yau's. The low energy degrees of freedom in the effective four-dimensional theory then correspond to Ricci flat deformations of metric of the 7-manifold. We will leave this investigation for future work. From a phenomenological point of view, it might be interesting to look for more general solutions in which the topology changes also with time. This may have implications for cosmology as well as other physics of the brane world scenario.

\section{Acknowledgments}

We would like to thank Eric Bergshoeff, Jan de Boer, Mirjam Cvetič, Frederik Denef, Ron Donagi, Michael Douglas, Antonella Grassi, Sergei Gukov, Shamit Kachru, Renata 
Kallosh, Chien-Hao Liu, David Morrison, Greg Moore, Burt Ovrut, Jaemo Park, Marco Serone, Stefan Vandoren and Edward Witten for useful discussions. The research of B.R.G. was partially supported by the DOE grant DE-FG02-92ER40699B. The research of G.S. was partially supported by the NSF grant PHY-97-22101 while he was at the C.N. Yang Institute for Theoretical Physics at Stony Brook where this work was completed, and was supported in part by the DOE grant FG02-95ER40893 and the School of Arts and Sciences Dean's fund at the University of Pennsylvania where this paper was written. K.S. is grateful for the extended hospitality of the Spinoza Institute at Utrecht and the C.N. Yang Institute for Theoretical Physics at Stony Brook.

\section{References}

[1] E. Witten, Nucl. Phys. B403 (1993) 159;

P.S. Aspinwall, B.R. Greene and D.R. Morrison, Phys. Lett. B303 (1993) 249; Nucl. Phys. B416 (1994) 414.

[2] B.R. Greene, D.R. Morrison and A. Strominger, Nucl. Phys. B451 (1995) 109.

[3] E. Witten, Nucl. Phys. B471, 195 (1996)

[4] P. Horava and E. Witten, Nucl. Phys. B460 (1996) 506;

E. Witten, Nucl. Phys. B471 (1996) 135;

P. Horava and E. Witten, Nucl. Phys. B475 (1996) 94.

[5] E. Witten, Nucl. Phys. B471, 135 (1996)

[6] A. Lukas, B.A. Ovrut, K.S. Stelle and D. Waldram, Phys. Rev. D59 (1999) 086001; See, for example, B.A. Ovrut, hep-th/9905115 and references therein.

[7] M. Bodner, A. C. Cadavid and S. Ferrara, supergravity Class. Quant. Grav. 8, 789 (1991).

[8] A. C. Cadavid, A. Ceresole, R. D'Auria and S. Ferrara, Phys. Lett. B357, 76 (1995)

[9] K. Behrndt and S. Gukov, Nucl. Phys. B580 (2000) 225.

[10] N. Arkani-Hamed, S. Dimopoulos and G. Dvali, Phys. Lett. B429 (1998) 263; I. Antoniadis, N. Arkani-Hamed, S. Dimopoulos and G. Dvali, Phys. Lett. B436 (1998) 257.

[11] G. Shiu and S.-H.H. Tye, Phys. Rev. D58 (1998) 106007.

[12] Z. Kakushadze and S.-H.H. Tye, Nucl. Phys. B548 (1999) 180.

[13] L. Randall and R. Sundrum, Phys. Rev. Lett 83 (1999) 4690.

[14] B. R. Greene, K. Schalm and G. Shiu, Nucl. Phys. B584 (2000) 480. 
[15] G. Tian and S. T. Yau, in San Diego 1986, Proc., Mathematical aspects of String Theory 543-559.

[16] E. Witten, Nucl. Phys. B463 (1996) 383.

[17] D.R. Morrison and C. Vafa, Nucl. Phys. B473 (1996) 74, Nucl. Phys. B476 (1996) 437.

[18] J. Louis, J. Sonnenschein, S. Theisen and S. Yankielowicz, Nucl. Phys. B480 (1996) 185.

[19] P. Candelas, A. Font, S. Katz and D.R. Morrison, Nucl. Phys. B429 (1994) 626.

[20] A. Chou, R. Kallosh, J. Rahmfeld, S.-J. Rey, M. Shmakova, W.K. Wong, Nucl. Phys. B508 (1997) 147;

[21] I. Gaida, S. Mahapatra, T. Mohaupt and W.A. Sabra, Class. Quant. Grav. 16 (1999) 419.

[22] K. Behrndt, D. Lust and W. A. Sabra, Phys. Lett. B418, 303 (1998)

[23] E. Bergshoeff, R. Kallosh and A. Van Proeyen, hep-th/0007044.

[24] M. J. Duff, R. Minasian and E. Witten, Nucl. Phys. B465, 413 (1996)

[25] C. Liu, hep-th/9801175.

[26] S. Ferrara, R. Kallosh and A. Strominger, Phys. Rev. D52 (1995) 5412.

[27] S. Ferrara and R. Kallosh, Phys. Rev. D54, 1514 (1996)

[28] S. Ferrara and R. Kallosh, Phys. Rev. D54, 1525 (1996)

[29] B. R. Greene, talk at Strings 2000, University of Michigan, Ann Arbor, http://feynman.physics.lsa.umich.edu/cgi-bin/s2ktalk.cgi?greene 